\title{
FOTOCATÁLISIS HETEROGÉNEA PARA EL TRATAMIENTO DE AGUAS RESIDUALES GENERADAS EN EL BAÑO DEL GANADO
}

\author{
HETEROGENEOUS PHOTOCATALYSIS FOR THE TREATMENT \\ OF WASTEWATER FROM CATTLE BATH
}

\begin{abstract}
${ }^{1}$ Arnulfo Estrada-Martínez, 2 José Ortega-Ruiz, ${ }^{3}$ Iván Urango-Cardenas, ${ }^{4}$ German Enamorado-Montes, ${ }^{5}$ José Marrugo-Negrete

1,2,3,4 y 5 Universidad de Córdoba, Colombia

1 aresma74@gmail.com; 2 jortegaruiz@correo.unicordoba.edu.co;

${ }^{3}$ ivanurango@correo.unicordoba.edu.co; ${ }^{4}$ genamoradomontes@correo.unicordoba.edu.co;

5 jmarrugo@correo.unicordoba.edu.co
\end{abstract}

\section{RESUMEN}

Las actividades ganaderas, además de utilizar grandes volúmenes de agua, generan una alta carga de contaminantes, especialmente de tipo orgánico, los cuales llegan directamente a las fuentes de agua natural alterando la calidad de las mismas. Debido a lo anterior, el objetivo del siguiente trabajo fue encontrar las condiciones óptimas de operación de un sistema de fotocatálisis heterogénea con $\mathrm{TiO}_{2}$ mediada con luz solar para el tratamiento de aguas residuales provenientes del baño de ganado; para ello se especificaron dos niveles de $\mathrm{pH}(5,0$ y 7,0$)$ y dos niveles de concentración de $\mathrm{TiO}_{2}(0,4$ y $0,6 \mathrm{~g} / \mathrm{L})$. En cada tratamiento se recircularon $40 \mathrm{~L}$ del agua residual en un colector parabólico compuesto solar durante 240 minutos. La eficiencia del sistema y la cinética de degradación se determinaron en términos de la demanda química de oxígeno (DQO). Las remociones de DQO en los tratamientos fueron mayores al $50 \%$, mientras que el diseño experimental Taguchi y la mejor relación señal/ruido $\left(R_{S / R}\right)$ mostraron que las condiciones óptimas de operación se obtienen para un $\mathrm{pH}$ igual a 5,0 y una concentración de $\mathrm{TiO}_{2}$ de $0,6 \mathrm{~g} / \mathrm{L}$ alcanzando remociones del 83,2 \% La cinética de degradación fue de primer orden con mayores velocidades de reacción para las mayores cantidades de UV

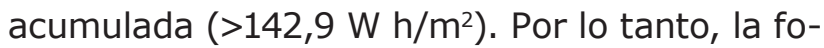
tocatálisis heterogénea con $\mathrm{TiO}_{2}$ mediada por luz solar resulta ser un tratamiento viable para la remoción de contaminantes de tipo orgánico en efluentes afectados por el desarrollo de actividades ganaderas.

Palabras claves: Contaminantes Orgánicos; Ganadería; Tratamiento del agua, Fotocatálisis heterogénea 


\section{ABSTRACT}

Livestock activities, in addition to using large volumes of water generate a high load of pollutants, especially of organic type, which arrive directly at natural water sources altering their quality. Due to the above, the aim of the following research was to find the optimal operating conditions of a heterogeneous photocatalysis system with $\mathrm{TiO}_{2}$ mediated with sunlight for the treatment of wastewater from the cattle bath; For this purpose, two pH levels (5.0 and 7.0) and two levels of $\mathrm{TiO} 2$ concentration $(0.4$ and $0.6 \mathrm{~g} / \mathrm{L})$ were specified. In each treatment, $40 \mathrm{~L}$ of the wastewater was recirculated in a solar composite parabolic collector for 240 minutes. The efficiency of the system and the kinetics of degradation were determined in terms of chemical oxygen demand (COD). The COD removals in the treatments were greater than $50 \%$, while the Taguchi experimental design and the best signal/noise ratio $\left(R_{S / R}\right)$ showed that the optimal operating conditions are obtained for a $\mathrm{pH}$ equal to 5.0 and a concentration of $\mathrm{TiO} 2$ of $0.6 \mathrm{~g} / \mathrm{L}$ reaching removals of $83.2 \%$. The degradation kinetics was of first order with higher reaction rates for the highest amounts of accumulated UV (> 142.9 W h/m2). Therefore, heterogeneous photocatalysis with $\mathrm{TiO} 2$ mediated by sunlight turns out to be a viable treatment for the removal of organic pollutants in effluents affected by the development of livestock activities.

Keywords: Organic Pollution; Livestock; Pesticides; Water treatment; heterogeneous photocatalysis.

\section{INTRODUCCIÓN}

En Latinoamérica, los problemas de índole parasitaria afectan las explotaciones ganaderas causando gran impacto sanitario y económico en dicha actividad, lo que motiva la utilización de pesticidas como antiparasitarios de uso interno y externo (López et al., 2009; Martínez y Cruz, 2009). A pesar de que estos pesticidas representan un beneficio en la eliminación y prevención de estos parásitos, su impacto ambiental negativo requiere estrategias efectivas de manejo y tratamiento de sus residuos. En la actualidad, las granjas ganaderas no cuentan con un tratamiento adecuado para las aguas residuales que se generan como producto de sus actividades y en las que se han detectado residuos de plaguicidas, tales como la cipermetrina y algunos sinérgicos como el Butoxido de Piperonilo, además de altas cargas de materia orgánica (Marrugo-Negrete et al., 2016), lo cual se refleja en altos valores de parámetros fisicoquímicos como la DQO, que se relaciona directamente con la cantidad de compuestos orgánicos oxidables en una muestra liquida.

La fotocatálisis heterogénea es una tecnología avanzada de oxidación que permite la degradación y la mineralización de gran variedad de compuestos orgánicos (Garcés et al., 2004). El proceso se basa en la excitación de un fotocatalizador sólido, normalmente semiconductor de banda ancha, sumergido en una solución, el cual, mediante la absorción de energía radiante (visible o UV), origina reacciones simultáneas de oxidación y reducción en diferentes zonas de la región interfacial (Peiró, 2003). El catalizador más utilizado es la forma cristalina anatasa del dióxido de titanio $\left(\mathrm{TiO}_{2}\right)$, por ser muy activo para la fotocatálisis, económico, inerte y resistente a la fotocorrosión. Una de las ventajas de este proceso es su capacidad de destruir prácticamente cualquier tipo de sustancia 
orgánica, incluidas mezclas complejas. En este sentido, es capaz de descomponer incluso sustancias cuyo tratamiento, empleando otros métodos, es difícil o peligroso, tales como las dioxinas, bifenilos policlorados, pesticidas, colorantes o fármacos (Colina-Márquez y CastillaCaballero, 2013; Dasary et al., 2010; Son et al., 2009; Samara et al., 2015; Raza et al., 2015). Por lo anterior, esta tecnología actualmente se ha implementado para la degradación fotocatalítica de contaminantes emergentes usados en el baño del ganado, obteniendo resultados satisfactorios (Barrios y Yepes, 2010).

La fuente de energía del proceso fotocatalítico es la radiación UV, la región caribe donde se encuentra el departamento de Córdoba en Colombia recibe entre 1.730 y $2.000 \mathrm{~kW} \cdot \mathrm{m}^{-2}$. año-1, comparado con los lugares donde se reciben las mayores radiaciones del mundo $\left(2.500 \mathrm{~kW} \cdot \mathrm{m}^{-2}\right.$.año $\left.{ }^{-1}\right)$, presenta la ventaja de que la variación mensual de la radiación global frente a la media anual es pequeña (Rodríguez, 2008), la implementación de tecnologías que aprovechen la radiación solar en esta región podrían ser favorables. Por lo anterior, este trabajo se propone optimizar un sistema fotocatalítico con $\mathrm{TiO}_{2}$ como catalizador y luz solar como fuente de UV para el tratamiento de aguas contaminadas con compuestos orgánicos tales como plaguicidas provenientes del baño de ganado, utilizando como parámetro de monitoreo la medición de DQO, con el fin de brindar un tratamiento adecuado a los residuos generados durante las actividades agrícolas.

\section{MATERIALES Y MÉTODOS}

\section{Recolección de la muestra:}

El agua residual se obtuvo de una finca ganadera del departamento de Córdoba (Colombia), situada en zona rural del Municipio de Montería. Esta Finca utiliza el baño de los animales con bomba de espalda como método para el control de ectoparásitos. En este método se adiciona un acaricida a una dosis sugerida por el fabricante $(1,0 \mathrm{~mL}$ del producto comercial por cada litro de agua). La muestra fue obtenida después del lavado de los corrales desde los canales de recolección del establecimiento, y se almacenó en recipientes plásticos de $250 \mathrm{~L}$ hasta su transporte hacia la Universidad de Córdoba, donde se encontraba el sistema de fotocatálisis heterogénea (Figura 1). Con el fin de remover los sólidos y disminuir la turbidez, la muestra fue pretratada con un filtro lento de arena de tipo descendente-ascendente, según lo descrito por Durango et al., (2014).

\section{Sistema piloto de fotocatálisis heterogénea:}

El sistema consistió de un colector parabólico compuesto (CPC) como el descrito por Colina-Márquez et al., (2009), conformado por 10 tubos de borosilicato (Duran $®$ de Schott) con $1,2 \mathrm{~m}$ de longitud, $32,0 \mathrm{~mm}$ de diámetro externo y $1,4 \mathrm{~mm}$ de espesor, reflectores parabólicos de aluminio anodizado $(1,1 \mathrm{~m}$ de longitud). Todo el conjunto se ubicó sobre un soporte de hierro de dimensiones 2,95 × 0,60 $\times 0,95 \mathrm{~m}$. El colector CPC fue conectado a un tanque plástico de 70 L que contenía el agua residual, mientras que una electrobomba de $0,5 \mathrm{hp}$ con un caudal aproximado de $30 \mathrm{~L} / \mathrm{min}$ permitió el flujo del agua en el sistema. El volumen irradiado es de 8,23 L, y el área expuesta de 1,21 $\mathrm{m}^{2}$. El catalizador empleado fue $\mathrm{TiO}_{2}$ Degussa $\mathrm{P}-25$ con un área superficial específica de $50 \mathrm{~m}^{2} / \mathrm{g}$; densidad de $4,1 \mathrm{~g} / \mathrm{cm}$; absortividad específica $k$ de 174,74 $\mathrm{m}^{2} / \mathrm{kg}$; coeficiente de dispersión $\sigma$ de 1295,75 m²/ $\mathrm{kg}$. La radiación UV solar se midió con un sensor Acadus 85-PLS (rango entre 300 y 400 nm), las lecturas se registraron con un monitor FMC-3000 de Design Instruments. Un esquema general se muestra en la figura 1. 


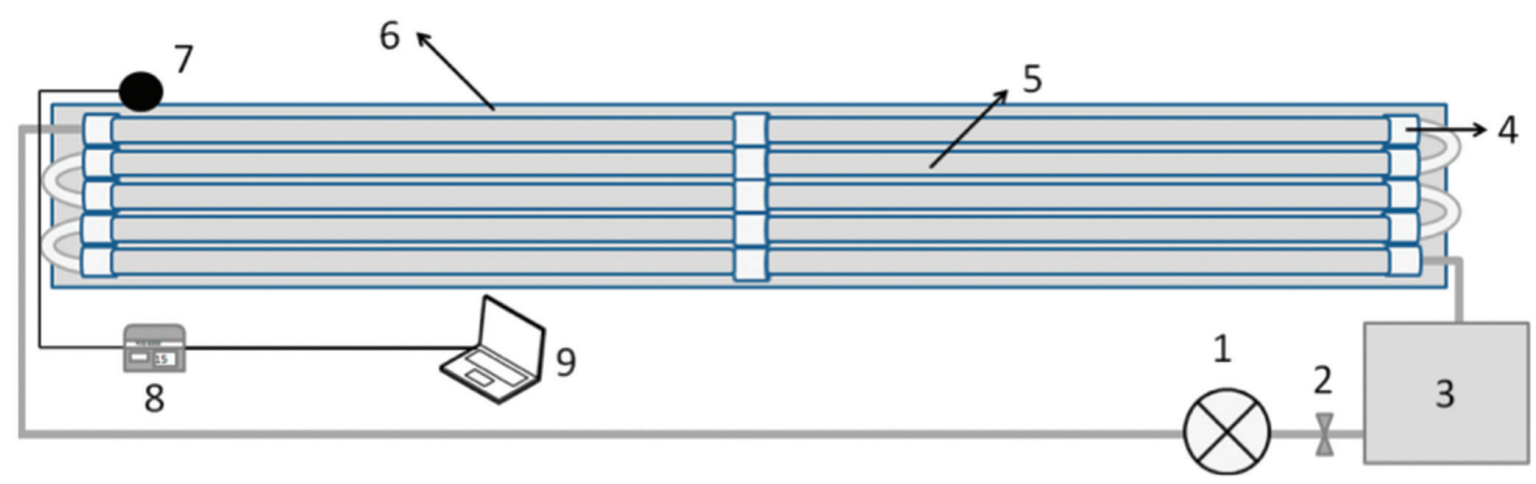

Figura 1. Esquema del sistema fotocatalítico empleado para el experimento. 1) Electrobomba; 2) llave de paso 3) tanque de almacenamiento de $70 \mathrm{~L}$; 4) conexiones de 1 in en PVC; 5) tubos de borosilicato; 6) reflectores de aluminio anodizado; 7) sonda de medición; 8) monitor FMC-3000;

9) Computador con el software Proasis Dcswin 3.57.

\section{Operación del sistema fotocatalítico:}

Los experimentos se realizaron en los meses de abril y mayo, en el Campus de la Universidad de Córdoba, Montería, Colombia ( $8^{\circ} 47^{\prime}$ 21.43" Norte y $75^{\circ} 51^{\prime} 30.64^{\prime \prime}$ Oeste). La zona se caracteriza por presentar entre 6 y 8 horas diarias de radiación solar durante todo el año (UPME-IDEAM, 2005). En cada ensayo, se recircularon $40 \mathrm{~L}$ de muestra filtrada durante 4 horas en el sistema fotocatalítico, desde las 10:00 horas hasta las 14:00 horas, estudios realizados en el mismo campus universitario destacan las más altas radiaciones durante este rango de horas (Mercado y Vergara, 2014). El pH inicial fue medido con una consola Thermo Scientific Orion 4 Star y un electrodo de $\mathrm{pH}$ de cuerpo epóxico 5107BNMD, para el ajuste de $\mathrm{pH}$ se emplearon soluciones $1 \mathrm{~N}$ de $\mathrm{HCl} \circ \mathrm{NaOH}$. Para todos los ensayos, el $\mathrm{TiO}_{2}$ adicionado fue recirculado durante 20 minutos en oscuridad, con el fin de lograr el equilibrio de adsorción en la superficie de la matriz.

\section{Determinación de Demanda Química de Oxígeno (DQO):}

Se retiró cualquier exceso de $\mathrm{TiO}_{2}$ mediante centrifugación durante 3 minutos a 2.500 rpm. Las muestras se analizaron por el método espectrofotométrico tras la digestión de las mismas con solución ácida bajo reflujo cerrado de acuerdo con el procedimiento estándar de análisis SM 5220D (APHA-AWWA-WEF, 2005).

\section{Eficiencia del sistema de fotocatálisis heterogénea:}

La eficiencia del sistema se expresó en términos de la remoción de DQO, calculada de acuerdo con la ecuación 1:

$$
\% \text { remoción } D Q O=\frac{C_{0}-C_{i}}{C_{0}} \times 100 \%
$$

Donde, $C_{0}$ es la concentración de DQO inicial de la muestra en $\mathrm{mg} / \mathrm{L}$ y $\mathrm{Ci}$ es la concentración de DQO en mg/L después de 240 minutos de tratamiento.

\section{Optimización de las condiciones de operación del sistema fotocatalítico:}

Para optimizar el sistema, se consideró la concentración de $\mathrm{TiO}_{2}$ y el pH inicial como variables controlables, y la cantidad de energía acumulada UV como variable no controlable o de ruido (tabla 1), el porcentaje de remoción de DQO fue la variable de respuesta. Se empleó el diseño experimental de Taguchi, el cual consistió de un arreglo ortogonal L8 $\left(2^{3}\right)$ para 8 corridas con una réplica. Debido a que 
el objetivo fue maximizar la remoción de DQO, identificando los valores óptimos de $\mathrm{pH}$ y concentración de $\mathrm{TiO}_{2}$ que brinden robustez al diseño. En cuanto a las variaciones en la energía UV acumulada, el criterio estadístico elegido fue "Mayor es mejor", en donde la $\mathrm{R}_{\mathrm{S} / \mathrm{R}}$ se calcula a partir de la ecuación 2, en la cual se tiene " $n$ " lecturas, ... . Niveles con mayor $R_{S / R}$ son más favorables para la degradación de DQO, indicando que el proceso es menos sensible a la variación de la radiación solar.

$$
\frac{S}{R}=-10 \log \left[\frac{1}{n} \sum Y_{i}^{-2}\right]
$$

Tabla 1. Factores experimentales del diseño Taguchi

\begin{tabular}{l|c|c|c|c}
\multirow{2}{*}{ Factores } & \multirow{2}{*}{ Unidades } & \multicolumn{1}{c}{ Tipo } & \multicolumn{2}{c}{ NIVELES } \\
\cline { 4 - 5 } & unidad & Controlable & 5,0 & 7,0 \\
\hline $\mathrm{A}: \mathrm{pH}$ & $\mathrm{g} / \mathrm{L}$ & Controlable & 0,4 & 0,6 \\
\hline $\mathrm{B}: \mathrm{TiO}_{2}$ & $\mathrm{~W} \cdot \mathrm{h} / \mathrm{m}^{2}$ & Ruido & $<142,9$ & $\geq 142,9$ \\
\hline $\mathrm{C}: \mathrm{UV}$ & & & & Alto \\
\hline
\end{tabular}

Los niveles de las variables $\mathrm{TiO}_{2}$ y $\mathrm{pH}$, fueron seleccionados de acuerdo con los resultados obtenidos por Malato et al., (2001). Para el $\mathrm{TiO}_{2}$ $(0,4$ y $0,6 \mathrm{~g} / \mathrm{L})$, concentraciones por debajo de $0,2 \mathrm{~g} / \mathrm{L}$ no presentan una buena área superficial para garantizar una adecuada adsorción $y$, por encima de $1,0 \mathrm{~g} / \mathrm{L}^{1}$, producen un efecto de apantallamiento que reduce la velocidad de reacción. Por otro lado, valores bajos de $\mathrm{pH}$ favorecen la eficiencia del proceso fotocatalítico, por lo que se consideró para el nivel bajo un $\mathrm{pH}$ de 5,0 y para el nivel alto un $\mathrm{pH}$ de 7,0 , cercano al pH natural de la muestra $(7,45)$.

Se trabajó con dos niveles para la variable ruido, obtenidos a partir de mediciones realizadas durante 15 días previos al inicio del experimento, en los que se registró la radiación solar acumulada para las 4 horas de trabajo previamente seleccionadas. Se establecieron días nublados y soleados de acuerdo con el promedio de energía UV acumulada. Se consideró, como nivel bajo, las radiaciones solares acumuladas menores que $142,9 \mathrm{~W} \mathrm{~h} / \mathrm{m}^{2}$ $\mathrm{y}$, como nivel alto, las mayores o iguales a $142,9 \mathrm{~W} \mathrm{~h} / \mathrm{m}^{2}$.

\section{CINÉTICA DE DEGRADACIÓN}

Para el estudio cinético se trabajó con las condiciones óptimas de operación arrojadas por el diseño experimental Taguchi. Se tomaron muestras a diferentes tiempos de operación del sistema: 0, 30, 60, 120, 180 y 240 minutos y se determinó la concentración de DQO. La ecuación 3 es una expresión no-lineal (exponencial) simplificada de la cinética de Langmuir-Hinshelwood, la cual describe de manera acertada la degradación de compuestos orgánicos en procesos de fotocatálisis heterogénea, reduciendo el error de la ecuación lineal (Asenjo et al., 2013).

$$
C=C_{0} \exp \left(-k_{a p p} t\right)
$$

Donde $C_{0}$ es la concentración inicial $(\mathrm{mg} / \mathrm{L})$, $C$ es la concentración $(\mathrm{mg} / \mathrm{L})$ en el tiempo $t$ (min) y $k_{\text {app }}$ es la constante de velocidad aparente $\left(\mathrm{min}^{-1}\right)$.

\section{Resultados y discusión}

La muestra de agua residual recolectada evidenció un alto contenido de DQO (1996,8 mg/L) y de fósforo total (31,05 mg/L), estas concentraciones son comunes en este tipo de muestras (Lim et al., 2012; Carvalho et al., 2013; Tak et al., 2015) y demuestran una urgente necesidad de tratamiento, dado que los valores de DQO superan lo establecido por la reglamentación Colombiana en la resolución 0631 de 2015 emitida por el ministerio de ambiente (500 mg/ $\mathrm{L} \mathrm{O}_{2}$ ) para vertimientos puntuales de aguas residuales de actividades ganaderas a cuerpos de agua superficiales. En la tabla 2 se 
observan las características del agua residual, se puede notar que, luego del pretratamiento por filtración, el agua permanece con una concentración elevada de DQO $(998,4 \mathrm{mg} / \mathrm{L})$.Por otro lado, el pH del agua filtrada se acerca a la neutralidad (seleccionado como el nivel alto para la variable $\mathrm{pH}$ ).

Tabla 2. Características del agua residual proveniente del baño de ganado antes y después del filtrado.

\begin{tabular}{l|l|l} 
& $\begin{array}{l}\text { Muestra } \\
\text { Inicial }\end{array}$ & $\begin{array}{l}\text { Muestra } \\
\text { Filtrada }\end{array}$ \\
\hline $\mathrm{pH}$ & $7,45 \pm 0,35$ & $7,15 \pm 0,39$ \\
\hline $\mathrm{DQO}, \mathrm{mg} / \mathrm{L}$ & $1996,8 \pm 15,5$ & $998,4 \pm 11,5$ \\
\hline Fósforo total, $\mathrm{mg} / \mathrm{L}$ & $31,05 \pm 0,94$ & $19,37 \pm 0,52$ \\
\hline $\begin{array}{l}\text { Sólidos suspendidos } \\
\text { totales, } \mathrm{mg} / \mathrm{L}\end{array}$ & $141,46 \pm 10,92$ & $75,10 \pm 8,35$ \\
\hline
\end{tabular}

* Los valores corresponden a promedios de triplicados de análisis

\section{Eficiencia del sistema fotocatalítico y condiciones óptimas de operación}

Los porcentajes más bajos de eliminación de DQO se presentaron para las radiaciones UV $<142,9 \mathrm{~W} \mathrm{~h} / \mathrm{m}^{2}$, con una concentración de $\mathrm{TiO}_{2}$ de $0,4 \mathrm{~g} / \mathrm{Ly} \mathrm{pH}$ de 7,0 (51,28 \%). Por su parte las mejores remociones se lograron a $\mathrm{pH}$ de 5,0 , concentración de $\mathrm{TiO}_{2}$ de $0,6 \mathrm{~g} / \mathrm{L}$ y UV acumulada alta, lográndose remociones superiores al 80 \% (tabla 3). En ensayos por fotocatálisis heterogénea de aguas contaminadas con compuestos orgánicos (Gil et al., 2006) se utilizaron procesos avanzados de oxidación como la fotocatálisis heterogénea y homogénea para la degradación de colorantes, evaluando los porcentajes de degradación de DQO como una de las variables respuestas y alcanzando valores superiores al 66 \% de degradación. Vásquez et al., (2010) evaluaron la degradación del plaguicida Clorotalonilo (400 mg/L) obteniendo un $95 \%$ de remoción tras cuatro horas de tratamiento a una concentración de
$\mathrm{TiO}_{2}$ de $0,5 \mathrm{~g} / \mathrm{L}$ y a pH 3,0. Arroyave y Garcés (2011) estudiaron la remoción del pesticida Mertect por fotocatálisis heterogénea con $\mathrm{TiO}_{2}$ bajo luz artificial UV, alcanzando remociones del 99,5 \% para una carga de catalizador de $0,5 \mathrm{~g} / \mathrm{L}$ y un $\mathrm{pH}$ de 5,0. Fenoll et al.,(2012), Reportaron remociones de hasta un $70 \%$ en la degradación de cinco plaguicidas triazínicos y tres cloroacetanilidas mediante fotocatálisis heterogénea con $\mathrm{TiO}_{2}$ y $\mathrm{ZnO}$, con cargas de catalizador entre 0 a $250 \mathrm{mg} / \mathrm{L}$, al incrementarse la concentración del catalizador las remociones fueron mayores, mientras que variaciones de $\mathrm{pH}$ en el rango de 6,0 a 9,0 no significaron mejorías en las eficiencias de remoción para tiempos de irradiación de 240 minutos.

Tabla 3. Resultados porcentaje de degradación de la DQO. Arreglo interno y externo, diseño experimental de Taguchi.

\begin{tabular}{l|c|c|c}
\multicolumn{2}{|c|}{ Arreglo interno } & \multicolumn{3}{|c}{ Arreglo externo } \\
\hline A: pH & B: $\left[\mathrm{TiO}_{2}\right]$ & C: UV Baja & C: UV Alta \\
\hline 5,0 & 0,4 & $74,81 \%$ & $80,32 \%$ \\
\hline 7,0 & 0,4 & $51,28 \%$ & $73,95 \%$ \\
\hline 5,0 & 0,6 & $76,94 \%$ & $83,16 \%$ \\
\hline 7,0 & 0,6 & $66,07 \%$ & $77,68 \%$ \\
\hline
\end{tabular}

Las condiciones óptimas de operación del sistema fueron calculadas estadísticamente mediante la respuesta de la $\mathrm{R}_{\mathrm{S} / \mathrm{R}^{\prime}}$ como se observa en la figura 2. Los niveles con mayor $R_{S / R}$ son los más favorables para la remoción de la DQO. Para el presente trabajó se encontró que un $\mathrm{pH}$ de $5,0\left(\mathrm{R}_{\mathrm{S} / \mathrm{R}}\right.$ de 37,92$)$ y una concentración de $\mathrm{TiO}_{2}$ de $0,6 \mathrm{~g} / \mathrm{L}\left(\mathrm{R}_{\mathrm{S} / \mathrm{R}}\right.$ de 37,58$)$, representan condiciones en las cuales se da la degradación del contaminante sin que el proceso se vea afectado significativamente por la variabilidad del factor ruido. Otros estudios también demuestran que la degradación de plaguicidas como la cipermetrina mediante fotocatálisis heterogénea usando $\mathrm{TiO}_{2}$, se 
ve favorecida a un pH ácido (Yao et al., 2007; Affam y Chaudhuri, 2013), bajo ciertas condiciones de $\mathrm{pH}$ las sustancias orgánicas pueden disociarse y adquirir una carga neta negativa, favoreciendo su adsorción hacia la superficie del catalizador cargada positivamente bajo estas condiciones (Valencia et al., 2011; Gao et al., 2011; Vineetha et al., 2013).

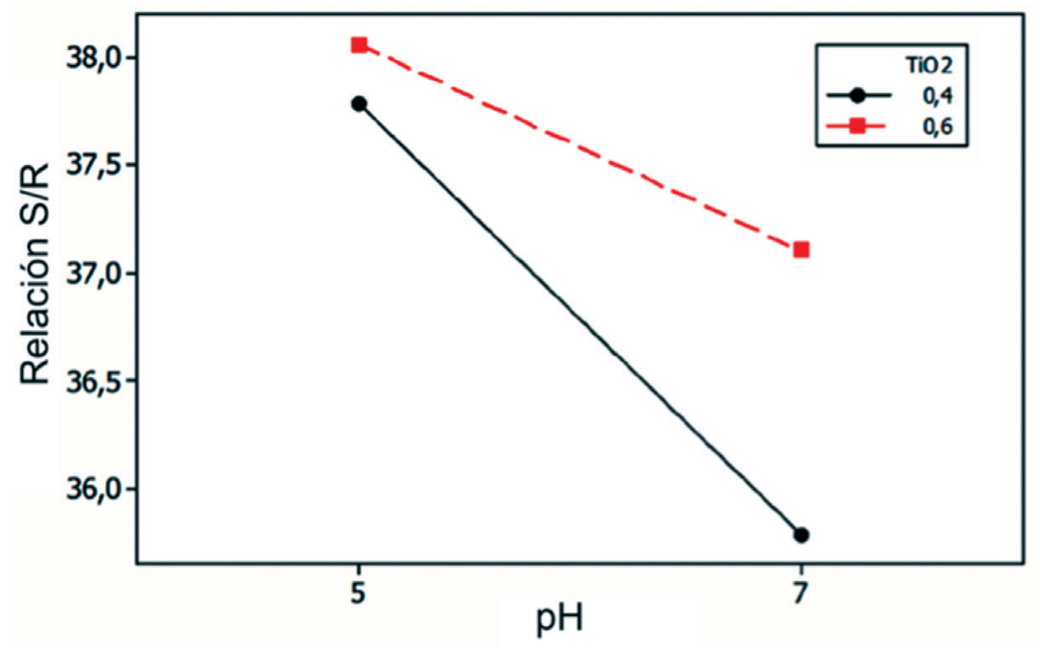

Figura 2. Interacción de las variables de estudio de acuerdo con relaciones S/R empleando el estadístico "más grande es mejor".

Efectos del pH y la concentración de $\mathrm{TiO}_{2}$ sobre la remoción:

Para el análisis estadístico ANOVA se consideró la radiación UV acumulada como el error experimental. En el ANOVA de la tabla 5 se aprecia que no existen diferencias estadísticamente significativas entre los tipos de tratamiento, tanto para los efectos como en su interacción, para un nivel de confianza del $95 \%$. El valor de la suma de cuadrados del error experimental resultó muy significativo, indicando que la radiación UV acumulada, a pesar de haber sido considerada como el factor ruido para el diseño experimental, representó un impacto muy considerable sobre el porcentaje de degradación del contaminante, siendo lo esperado, puesto que la cantidad de fotones absorbidos en el proceso depende directamente de la cantidad de energía radiante UV acumulada lo que aumenta las probabilidades de generación de pares electrón/huecos en la superficie del catalizador y de radicales $\mathrm{OH}$, lo cual aumenta las reacciones de oxidación del contaminante y por lo tanto, su degradación.

Tabla 5. Análisis de varianza para las remociones de DQO, sin considerar la radiación acumulada, representada en el error experimental.

\begin{tabular}{l|c|c|c|c|c}
\hline Fuente & Suma de Cuadrados & GI & \multicolumn{1}{c}{ Cuadrado Medio } & Razón-F & Valor-P \\
\hline $\mathrm{A}: \mathrm{pH}$ & 267,383 & 1 & 267,383 & 2,98 & 0,1594 \\
\hline $\mathrm{B}: \mathrm{TiO}_{2}$ & 68,9725 & 1 & 68,9725 & 0,77 & 0,4301 \\
\hline $\mathrm{AB}\left(\mathrm{pH}^{*} \mathrm{TiO}_{2}\right)$ & 22,9503 & 1 & 22,9503 & 0,26 & 0,6396 \\
\hline Error total & 358,885 & 4 & 89,7212 & & \\
\hline Total corregido & $\mathbf{7 1 8 , 1 9}$ & $\mathbf{7}$ & & & \\
\hline
\end{tabular}




\section{Cinética de degradación:}

El proceso de degradación fotocatalítica de la DQO en muestras reales de aguas residuales provenientes del baño de ganado se explica por una cinética de Langmuir-Hinshelwood de primer orden, puesto que los puntos experimentales se ajustan mejor a la ecuación exponencial, con una correlación de 0,9892 para radiación UV alta, y 0,9380 para radiación UV baja; mientras que la cinética de reacción de orden cero presentó un $\mathrm{R}$ de 0,9447 y 0,9350 para radiaciones acumuladas altas y bajas respectivamente, y la cinética de pseudo segundo orden fue la que mostró más bajos valores de correlación, 0,9074 y 0,8903. La cinética de primer orden de Langmuir-Hinshelwood intenta explicar las reacciones que ocurren en la superficie del catalizador, asumiendo que este tiene siempre la misma capacidad de adsorción y no considera las interacciones entre las moléculas absorbidas. Muchos estudios sobre procesos fotocatalíticos heterogéneos para la degradación de compuestos orgánicos, en muestras reales y sintéticas, se han estudiado y comprobado mediante este tipo de cinética (Son et al., 2009; Khan et al., 2010; Sahoo et al., 2012; Montazerozohori et al., 2012; Zhao et al., 2014; da Silva et al., 2015). Por lo anterior, se deduce que la velocidad de reacción en el porcentaje de degradación de la DQO depende, en gran medida, de la concentración inicial del plaguicida. El coeficiente de determinación $\mathrm{R}$ de 0,9892 indica que el 98,92 \% de la respuesta observada en la remoción de la DQO puede explicarse por el tiempo de exposición para UV alta, así mismo, el coeficiente de determinación $\mathrm{R}$ de 0,938 indica que el $93,8 \%$ de la respuesta observada en la remoción de la DQO puede explicarse por el tiempo de exposición para UV baja, como se muestra en la figura 3.

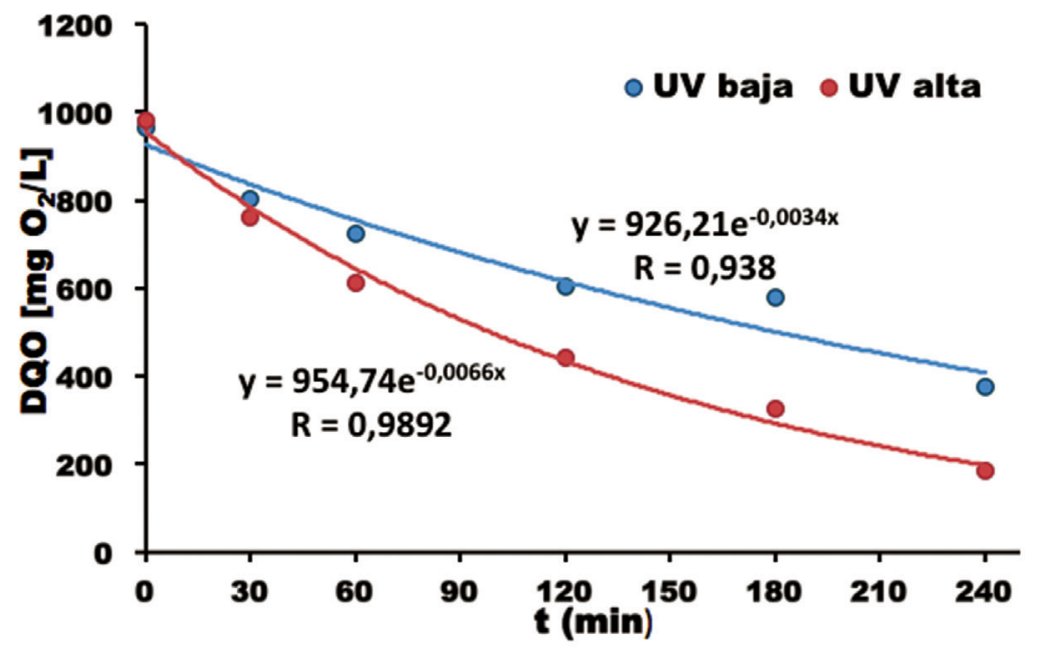

Figura 3. Cinética de primer orden para las condiciones óptimas encontradas pH 5.0 concentración de $\mathrm{TiO}_{2} 0.6 \mathrm{~g} \cdot \mathrm{L}^{-1}$ con energía UV acumulada alta y baja.

Las constantes de velocidad aparente $k_{\text {app }}$ fueron 0,0066 $\mathrm{min}^{-1}$ para radiación UV alta, y 0,0034 $\mathrm{min}^{-1}$ para radiación UV baja, indicando una mayor tasa de degradación durante los días soleados que durante días nublados. Lo anterior se explica debido a que durante los ensayos realizados con radicación UV acumulada baja aproximadamente un 50 \% de los 
fotones UV disponibles en la radiación solar se encuentran en el componente difuso (Giménez et al., 1999), limitando el aprovechamiento de los fotones disponibles para la generación del par - hueco $\mathrm{e}_{\mathrm{bv}}{ }^{-} \mathrm{y} \mathrm{h}_{\mathrm{bc}}{ }^{+}$. En la radiación acumulada alta, donde la disponibilidad de fotones genera suficientes radicales $\mathrm{OH}$, la cinética de degradación está determinada, principalmente, por la concentración del contaminante, duplicando el valor de la constante cinética aparente.

\section{CONCLUSIONES}

El tratamiento por fotocatálisis heterogénea en aguas residuales del baño de ganado permitió alcanzar porcentajes de remoción de DQO mayores al 80 \% en un tiempo de 4 horas, este proceso fue más eficiente para radiaciones acumuladas altas que para acumuladas bajas. A pesar de la necesidad de radiaciones adecuadas para la activación del catalizador, la radiación solar puede resultar una fuente de energía óptima para realizar procesos fotocatalíticos en el campus de la universidad de Córdoba y en fincas agropecuarias del departamento, puesto que la posición astronómica de la región permite presentar condiciones de radiación similares en todo el territorio, por lo tanto la fotocatálisis heterogénea con $\mathrm{TiO}_{2}$ mediada por luz solar puede ser un tratamiento viable para la remoción de la carga de DQO en efluentes contaminados en estas explotaciones ganaderas. El proceso fotocatalítico fue favorecido por un $\mathrm{pH}$ ácido, donde la diferencia de cargas entre el catalizador y el contaminante favorecían el proceso de adsorción, denotando la importancia del pH del medio. Igualmente, las condiciones óptimas del diseño Taguchi sugieren un nivel alto para la concentración de $\mathrm{TiO}_{2}$ si se quieren lograr las mejores eficiencias y lograr una mayor cantidad de pares electrón/huecos que aumenten la degradación del contaminante, sin sobrepasar concentraciones mayores a $1.0 \mathrm{~g} . \mathrm{L}^{-1}$.

\section{AGRADECIMIENTOS}

Los autores agradecen al Instituto Colombiano de Desarrollo de Ciencias y Tecnología "Francisco José Caldas" COLCIENCIAS y a la Universidad de Córdoba, Colombia, por el convenio No. 549-2012

\section{BIBLIOGRAFÍA}

American Public Health Association (APHA), American Water Works Association (AWWA), Water Environmental Federation (WEF) (2005). Standard Methods for the Examination of Water and Wastewater, 21st ed., Maryland, Port City Press.

Affam A. y Chaudhuri M. (2013). Degradation of pesticides chlorpyrifos, cypermethrin and chlorothalonil in aqueous solution by $\mathrm{TiO} 2$ photocatalysis. Journal of Environmental Management, volumen 130. pp. 160-165.

Arroyave J.A. y Garcés L.F (2011). Evaluación de la degradación del pesticida Mertect empleando procesos avanzados de oxidación (PAO). Producción + Limpia, volumen 6 (número 2). pp. 9-18.

Asenjo N., Santamaría R., Blanco C., Granda M., Álvarez P., Menéndes R. (2013). Correct use of the Langmuir-Hinshelwood equation for proving the absence of a synergy effect in the photocatalytic degradation of phenol on a suspended mixture of titania and activated carbon. Carbon, Volumen 55, 62-69.

Barrios Barrios J., Yepez Montes J. (2010). Evaluación del modelo de la cinética de degradación fotocatalítica solar en un reactor CPC de un pesticida usado en el baño de ganado. Trabajo de Grado Programa de Ingenieria Quimica - Universidad De Cartagena, p. 66.

Carvalho P., Araujo J., Mucha A., Basto M., Almeida C. (2013). Potential of constructed wetlands microcosms for the removal of veterinary pharmaceuticals from livestock wastewater. Bioresource Technology, volumen 134, pp. 412-416.

Colina-Marquez J., Machuca-Martinez F., Puma G. (2009). Photocatalytic Mineralization of Commercial Herbicides in a Pilot-Scale Solar CPC Reactor: Photoreactor Modeling and Reaction Kinetics Constants Independent of Radiation Field. Environmental Science \& Technology, volumen 43 (número 23), pp. 8953-8960. 
Colina-Márquez J. y Castilla-Caballero D. (2013) Mineralización fotocatalítica de agua residual contaminada con dicloxacilina comercial en un reactor solar CPC a escala piloto. Ingeniería y Competitividad, volumen 15 (número 1), 161-169.

da Silva S., Klauck C., Siqueira M., Bernardes A. (2015). Degradation of the commercial surfactant nonylphenol ethoxylate by advanced oxidation processes. Journal of Hazardous Materials, volumen 282, pp. 241-248.

Dasary S., Saloni J., Fletcher A., Anjaneyulu Y., Yu H. (2010). Photodegradation of Selected PCBs in the Presence of Nano-TiO2 as Catalyst and H2O2 as an Oxidant. International Journal of Environmental Research and Public Health, volumen 7 (número 11), pp. 3987-4001.

Durango J, Urango I, Pinedo J, Burgos S, Estrada A, Ortega J, Taboada R, Figueroa J, Marrugo J, Enamorado G. (2014). Evaluación un filtro lenta de arena, de tipo descendente-ascendente, para el tratamiento de efluentes ganaderos contaminados con cipermetrina. Memorias VII Seminario Internacional de Gestión Ambiental y II Seminario de Ciencias Ambientales Sue-Caribe, Santa Marta, Colombia, [consulta, 12 de noviembre de 2014]. Recuperado de : http://maestriaambiental.com/ maestria/seminarioii/pages/memorias.

Fenoll J., Hellin P., Martinez C., Flores P., Navarro S. (2012). Semiconductor-sensitized photodegradation of s-triazine and chloroacetanilide herbicides in leaching water using $\mathrm{TiO} 2$ and $\mathrm{ZnO}$ as catalyst under natural sunlight. Journal of Photochemistry and Photobiology a-Chemistry, volumen 238, pp. 81-87.

Gao M., Wang X., Guo M., Zhang M. (2011). Contrast on COD photo-degradation in coking wastewater catalyzed by TiO2 and TiO2-TiO2 nanorod arrays. Catalysis Today, volumen 174 (número 1) pp. 79-87.

Garcés L., Mejia E., Santamaria J. (2004). La fotocatálisis como alternativa para el tratamiento de aguas residuales. Revista Lasallista de Investigación, volumen 1, pp. 83-92.

Gil Pavas E., Quintero Olaya L., Rincón Uribe M., Rivera Agudelo D. (2006). Degradación de colorantes de aguas residuales empleando UV/TiO2/ H2O2/Fe2+. REVISTA Universidad EAFIT, Volumen 42 (numero 146), pp. 80-101

Giménez J., Curcó D., Queral M.A. (1999). Photocatalytic treatment of phenol and 2,4-dichlorophenol in a solar plant in the way to scaling-up.
Catalysis Today, volumen 54 (número 2-3) pp. 229-243.

Khan U., Benabderrazik N., Bourdelais A., Baden D., Rein K., Gardinali P., Arroyo L., O'Shea K. (2010). UV and solar TiO2 photocatalysis of brevetoxins (PbTXs). Toxicon, volumen 55 (número 5), pp. 1008-1016.

Lim S., Park W., Kim T., Shin I. (2012). Swine wastewater treatment using a unique sequence of ion exchange membranes and bioelectrochemical system. Bioresource Technology, volumen 118, pp. 163-169.

López G., Grissi C., Gómez J., Valencia L.A., González D. (2009). Evaluación de una mezcla de cipermetrina + clorpirifós sobre la garrapata rhipicephalus(boophilus) microplus en pruebas de campo y de laboratorio en el predio Esteban Jaramillo Román Gómez del Politécnico Colombiano de Marinilla, Antioquia. Revista CES Medicina Veterinaria y Zootecnia, volumen 4 (número 2), pp. 57-65.

Malato S., Blanco J., Estrada C., Bandala R. (2001). Degradación de plaguicidas, en Blesa M. Eliminación de contaminantes por fotocatálisis heterogénea. Buenos Aires, Red CYTED, pp. 269-281.

Marrugo-Negrete J., Ortega-Ruíz J., Navarro-Frómeta A., Enamorado-Montes G., Urango-Cárdenas I., Pinedo-Hernández J., Durango-Hernández J., Estrada-Martínez A. (2016). Remoción de cipermetrina presente en el baño de ganado utilizando humedales construidos. Corpoica Ciencia y Tecnologia Agropecuaria, volumen 17 (número 2), pp. 203-216.

Martínez I. y Cruz M. (2009). El uso de químicos veterinarios y agrícolas en la zona ganadera de Xico, centro de Veracruz, México y el posible impacto ambiental. Acta Zoológica Mexicana, volumen 25 (número 3), pp. 673-681.

Mercado T. y Vergara A. (2014). Bombeo de agua con energía solar fotovoltaica: una opción sostenible, en Memorias VII Seminario Internacional de Gestión Ambiental y II Seminario de Ciencias Ambientales Sue-Caribe, Santa Marta, Colombia, 2014 [consulta, 12 de noviembre de 2014]. Recuperado de: http://maestriaambiental.com/ maestria/seminarioii/pages/memorias.

Ministerio de Minas y Energía -Unidad de Planeación Minero Energética (UPME) y Ministerio de Ambiente, Vivienda y Desarrollo Territorial Instituto de Hidrología, Meteorología y Estudios Ambientales (IDEAM) (2005). Atlas de Radiación 
Solar de Colombia. Bogotá, Colombia. [consulta, 22 de febrero de 2015]. Recuperado de: http://www.si3ea.gov.co/Home/EnergiaSolar/tabid/74/language/en-US/Default.aspx

Montazerozohori M., Nasr-Esfahani M., Joohari S. (2012). Photocatalytic degradation of an organic dye in some aqueous buffer solutions using nano titanium dioxide: a kinetic study. Environment Protection Engineering, volumen 38 (número3), pp. 45-55.

Peiró Muñoz A.M. (2003). Nuevas aportaciones al desarrollo de metodologías en química verde: eliminación fotocatalítica de contaminantes fenólicos, preparación de fotocatalizadores mediante procesos químicos suaves. Tesis (doctorado en química), España, Universitat Autònoma de Barcelona, p. 228.

Raza W., Haque M., Muneer M., Fleisch M., Hakki A., Bahnemann D. (2015). Photocatalytic degradation of different chromophoric dyes in aqueous phase using La and Mo doped TiO2 hybrid carbon spheres. Journal of Alloys and Compounds, volumen 632, pp. 837-844.

Rodríguez H. (2008). Desarrollo de la energía solar en Colombia y sus perspectivas. Revista de Ingeniería, (número 28) 83-89.

Sahoo C., Gupta A., Pillai I. (2012). Heterogeneous photocatalysis of real textile wastewater: Evaluation of reaction kinetics and characterization. Journal of Environmental Science and Health Part a-Toxic/hazardous Substances \& Environmental Engineering, volumen 47 (número 13), pp. 2109-2119.

Samara F., Jermani E., Kanan S.M. (2015). Photocatalytic UV-degradation of 2,3,7,8-tetrachlorodibenzo-p-dioxin (TCDD) in the presence of silver doped zeolite. Arabian Journal of Chemistry, doi:10.1016/j.arabjc.2014.12.009.
Son H., Ko G., Zoh K. (2009). Kinetics and mechanism of photolysis and TiO2 photocatalysis of triclosan. Journal of Hazardous Materials, volumen 166 (número 2-3), pp. 954-960.

Tak B.-y. Tak B.-s. Kim Y.-j. Park Y.-j. Yoon Y.-h. Min G.-h. (2015). Optimization of color and COD removal from livestock wastewater by electrocoagulation process: Application of Box-Behnken design (BBD). Journal of Industrial and Engineering Chemistry, doi:10.1016/j.jiec.2015.03.008.

Valencia S.H., Marín J.M., Restrepo G.M. (2011). Effect of $\mathrm{pH}$ on the photocatalytic degradation of natural organic matter. Información Tecnológica, volumen 22 (número 5), pp. 57-66.

Vasquez E., Penuela G., Agudelo S. (2010). Chlorothalonil photodegradation study using Fenton and photocathalysis techniques with titanium dioxide by means of solar radiation. Revista Facultad De Ingenieria-Universidad De Antioquia, (número 51), pp. 105-113.

Vineetha M., Matheswaran M., Sheeba K. (2013). Photocatalytic colour and COD removal in the distillery effluent by solar radiation. Solar Energy, volumen 91, pp. 368-373.

Yao B., Wang L., Wang C., Wang Y., Zhao G. (2007). Preparation and performances of RUO2/TiO2 films photocatalyst supported on float pearls. Chinese Journal of Chemical Physics, volumen 20 (número 6), pp. 789-795.

Zhao C., Pelaez M., Dionysiou D., Pillai S., Byrne J., O'Shea K. (2014). UV and visible light activated TiO2 photocatalysis of 6-hydroxymethyl uracil, a model compound for the potent cyanotoxin cylindrospermopsin. Catalysis Today, volumen 224, pp. 70-76.
Conflicto de Intereses Los autores declaran no tener ningún conflicto de intereses

\section{(@) $\odot(0)$}


